\title{
On the Beamforming Optimality Range in TIMO Channels with Common and Individual Input Power Constraints
}

\author{
Saad Al-Ahmadi and Halim Yanikomeroglu, Member, IEEE
}

\begin{abstract}
In this letter, the effect of the input power constraint on the beamforming optimality range in Gaussian two-input multiple-output (TIMO) channels is investigated. The obtained expressions, using standard Lagrangian formulation, determine explicitly the range of the input signal-to-noise ratio (SNR) for which beamforming (rank-1 signaling) is optimal for TIMO channels for both the common power constraint and the individual power constraint cases. Moreover, the obtained results are extended to random TIMO channels, with channel state information at the receiver only, using the Jensen's upper bound on the mutual information.
\end{abstract}

Index Terms-MIMO channels, mutual information, input covariance matrix, beamforming, common power constraint, individual power constraints.

\section{INTRODUCTION}

I $\mathrm{N}$ the design of the optimal transmission schemes for Gaussian multiple-input multiple-output (MIMO) channels, the range of optimality of beamforming (rank-1 signaling) is of relevance since scalar coding can be used to achieve the capacity $([1,2]$ and references therein). Moreover, the introduction of distributed MIMO systems [3, 4] and MIMOOFDM (orthogonal frequency-division multiplexing) systems [5] has motivated the research on the optimal input covariance for MIMO channels with individual power constraints. In this letter, the optimal (capacity-achieving) input covariance matrix is re-derived for Gaussian two input multi-output (TIMO) channels, using the standard Lagrangian approach revealing the explicit relation between the optimal input correlations and the input power constraint as well as the channel inner product matrix. Moreover, the implications of the obtained results to the range of optimality of beamforming in TIMO channels with individual power constraints are stated. Finally, these results are extended to random channels using Jensen's upper bound on mutual information.

In the letter, uppercase letters denote deterministic matrices and bold-faced uppercase letters denote random matrices. For vectors, bold-faced lowercase letters are used for both deterministic and random vectors where the distinction is assumed to be clear context-wise. The determinant, trace, adjoint and Hermitian of a matrix $A$ are denoted as $\operatorname{det}[A]$, $\operatorname{tr}(A), \operatorname{Adj}(A)$ and $A^{H}$, respectively; and $A \succeq 0$ denotes a

Paper approved by D. L. Goeckel, the Editor for Space-Time and OFDM of the IEEE Communications Society. Manuscript received February 19, 2009; revised December 15, 2009 and March 24, 2010.

S. Al-Ahmadi's work was supported by King Fahd University of Petroleum and Minerals, Saudi Arabia.

S. Al-Ahmadi is currently with the Department of Electrical Engineering, King Fahd University of Petroleum and Minerals, Saudi Arabia (e-mail: ahmadisa@kfupm.edu.sa).

H. Yanikomeroglu is with the Department of Systems and Computer Engineering, Carleton University, Ottawa, Canada (e-mail: halim@sce.carleton.ca).

Digital Object Identifier 10.1109/TCOMM.2011.010411.090022 positive semi-definite matrix. For a complex number $z$, the conjugate of $z$ and the real and imaginary parts of $z$ are denoted by $z^{*}, \Re(z)$, and $\Im(z)$, respectively.

\section{Deterministic Channel MATRIX}

\section{A. TIMO Channels with a Common Power Constraint}

The channel capacity of a MIMO channel with $L$ transmit and $M$ receive elements for a deterministic channel matrix $H \in \mathcal{C}^{M \times L}$ can be expressed as [6]

$$
\begin{gathered}
C=\max _{Q \succeq 0, \operatorname{tr}(Q)=P} \operatorname{logdet}\left[I_{M}+\frac{1}{\sigma_{n}^{2}} H Q H^{H}\right] \\
=\max _{Q \succeq 0, \operatorname{tr}(Q)=P} \operatorname{logdet}\left[I_{L}+\frac{1}{\sigma_{n}^{2}} Q H^{H} H\right],
\end{gathered}
$$

where $P$ is the total power constraint, $Q \in \mathcal{C}^{L \times L}$ is the positive semi-definite covariance matrix of the proper complex Gaussian input vector [7, Theorem 2]. In (1), $I_{M}$ denotes the $M \times M$ identity matrix and $\sigma_{n}^{2}$ is the variance of the complex Gaussian noise at each receive element. In subsequent derivations $\sigma_{n}^{2}$ is set to unity for notational simplicity. For a TIMO channel with a common power constraint, the expression in (1) reduces to

$$
C=\max _{Q \succeq 0, \operatorname{tr}(Q)=P} \operatorname{logdet}\left[I_{2}+Q H^{H} H\right] .
$$

The optimization problem in (1) and (2), and hence the channel capacity was characterized in [6] by considering the corresponding set of the orthogonal channels, obtained using the singular value decomposition (SVD) approach, and then applying the water-filling power allocation algorithm over these channels. However, in here we will consider the solution of (2) using the standard Lagrangian approach. In this regard, we may express the input covariance matrix of the input complex Gaussian vector as

$$
Q=\left[\begin{array}{cc}
\sigma_{1}^{2} & \rho_{12} \sigma_{1} \sigma_{2} \\
\rho_{21} \sigma_{1} \sigma_{2} & \sigma_{2}^{2}
\end{array}\right] .
$$

It can be shown using the solution proposed in Appendix A that we may express the optimal input correlation coefficient as

$$
\begin{gathered}
\rho_{12}=\frac{\mathbf{h}_{1}^{H} \mathbf{h}_{2}}{\sqrt{\frac{P^{2}}{4}-\Delta^{2}}\left[\left|\mathbf{h}_{1}\right|^{2}\left|\mathbf{h}_{2}\right|^{2}-\left|\mathbf{h}_{1}^{H} \mathbf{h}_{2}\right|^{2}\right]+\mu_{1}}, \\
\text { for }\left|\rho_{12}\right|^{2} \leq 1, \Delta \neq \frac{P}{2}, \text { and } \mathbf{h}_{1}^{H} \mathbf{h}_{2} \neq 0,
\end{gathered}
$$

where $\mathbf{h}_{1}$ and $\mathbf{h}_{2}$ denote the first and second columns of the channel matrix, respectively, and $\Delta$ denotes the disparity between the allocated powers as $\sigma_{1}^{2}=\frac{P}{2}+\Delta$ and $\sigma_{2}^{2}=\frac{P}{2}-\Delta$. 
Furthermore, the minimum input signal-to-noise ratio (SNR) below which beamforming is optimal is given as

$$
\sqrt{\frac{P^{2}}{4}-\Delta^{2}} \leq \frac{\left|\mathbf{h}_{1}^{H} \mathbf{h}_{2}\right|}{\left[\left|\mathbf{h}_{1}\right|^{2}\left|\mathbf{h}_{2}\right|^{2}-\left|\mathbf{h}_{1}^{H} \mathbf{h}_{2}\right|^{2}\right]} .
$$

For MIMO channels with a common power constraint, substituting $\Delta=\Delta_{1}$ as given in (23) in Appendix A leads to

$$
P \leq \sqrt{\frac{4\left|\mathbf{h}_{1}^{H} \mathbf{h}_{2}\right|^{2}+\left(\left|\mathbf{h}_{2}\right|^{2}-\left|\mathbf{h}_{1}\right|^{2}\right)^{2}}{\left[\left|\mathbf{h}_{1}\right|^{2}\left|\mathbf{h}_{2}\right|^{2}-\left|\mathbf{h}_{1}^{H} \mathbf{h}_{2}\right|^{2}\right]^{2}}} .
$$

The same expression can be obtained using the SVD approach since the minimum normalized power can be expressed as $P \leq \frac{1}{\lambda_{2}}-\frac{1}{\lambda_{1}}$ where $\lambda_{1}$ and $\lambda_{2}$ denote the eigenvalues of $H^{H} H[8]$.

\section{B. TIMO Channels with Individual Power Constraints}

The problem of determining the capacity of MIMO channels with individual "elemental" power constraints arises in (i) collocated MIMO systems where each antenna is equipped with its own power amplifier, (ii) distributed MIMO systems where each remote antenna has its own power constraint, (iii) MIMO-OFDM systems where it is preferable to use uniform power allocation across the transmit antennas, and (iv) digital subscriber lines with individual power constraints per modem. In general, for a MIMO channel with individual power constraints, the channel capacity can be expressed as

$$
C=\max _{Q \succeq 0, Q_{i i}=p_{i}} \log \operatorname{det}\left[I_{L}+Q H^{H} H\right]
$$

where $Q_{i i}$ denotes the $i$ th diagonal element of $Q$ and $\sum_{i=1}^{L} p_{i}=$ $P$. So, for TIMO channels with individual power constraints, the capacity optimization problem is similar to the one in (19) but with the diagonal elements of $Q$ being predetermined; hence the optimal input correlation coefficient, analogous to (4), can be expressed as

$$
\begin{aligned}
& \rho_{12}=\frac{\mathbf{h}_{1}^{H} \mathbf{h}_{2}}{\sqrt{\frac{P^{2}}{4}-\Delta_{2}^{2}}\left[\left|\mathbf{h}_{1}\right|^{2}\left|\mathbf{h}_{2}\right|^{2}-\left|\mathbf{h}_{1}^{H} \mathbf{h}_{2}\right|^{2}\right]+\mu_{2}}, \\
& \text { for } 0<\Delta_{2}<\frac{P}{2} \text { and } \mathbf{h}_{1}^{H} \mathbf{h}_{2} \neq 0,
\end{aligned}
$$

where again $\Delta_{2}$ denotes the difference in power allocation such that $\sigma_{1}^{2}=\frac{P}{2}+\Delta_{2}, \sigma_{2}^{2}=\frac{P}{2}-\Delta_{2}$, and $\mu_{2}$ has the identical expression as of $\mu_{1}$.

Proposition 1: The range of the normalized input SNR for which rank-1 signaling is optimal (capacity-achieving) for correlated TIMO channels with individual power constraints is given as

$$
\begin{aligned}
& 0<P \leq 2 \sqrt{\frac{\left|\mathbf{h}_{1}^{H} \mathbf{h}_{2}\right|^{2}}{\left[\left|\mathbf{h}_{1}\right|^{2}\left|\mathbf{h}_{2}\right|^{2}-\left|\mathbf{h}_{1}^{H} \mathbf{h}_{2}\right|^{2}\right]^{2}}+\Delta_{2}^{2}}, \\
& \text { for } 0<\Delta_{2}<\frac{P}{2} \text { and } \mathbf{h}_{1}^{H} \mathbf{h}_{2} \neq 0 .
\end{aligned}
$$

Proof: The proof is obtained in a straightforward manner using the expression in (8) and the fact that $\mu_{2}>0$ for $\left|\rho_{12}\right|^{2}=1$.
Remark: For the case $L=3$, the optimization problem can be expanded using the expression in (7). However, the condition for $Q$ to be semi-positive definite is more involved for $L=3$. Again using Sylvester's criterion for positive semi-definiteness [9], the non-negativity condition for the principal minors can be expressed as

$$
\begin{gathered}
\sigma_{1}^{2} \geq 0, \sigma_{2}^{2} \geq 0, \sigma_{3}^{2} \geq 0 \\
\left|\rho_{12}\right|^{2} \leq 1,\left|\rho_{13}\right|^{2} \leq 1,\left|\rho_{23}\right|^{2} \leq 1, \\
\sigma_{1}^{2} \sigma_{2}^{2} \sigma_{3}^{2}\left[1+2 \Re\left[\rho_{12} \rho_{23} \rho_{31}\right]-\left|\rho_{12}\right|^{2}-\left|\rho_{31}\right|^{2}-\left|\rho_{23}\right|^{2}\right] \geq 0 .
\end{gathered}
$$

Now, since the allocated powers are non-zero for a MIMO channel with individual power constraints, the problem of finding the rank of $Q$, and hence the rank-1 case, reduces to computing the corresponding optimal input correlation coefficients and determining the corresponding rank. Using the fact that for a non-singular matrix $A$,

$$
\frac{\partial[\operatorname{det}(A)]}{\partial x}=\operatorname{tr}\left(\operatorname{Adj}(A) \frac{\partial A}{\partial x}\right),
$$

the KKT conditions needed to solve for the optimal correlation coefficients can be expressed as

$$
\begin{aligned}
& c_{21} \sigma_{1} \sigma_{2}\left|\mathbf{h}_{1}\right|^{2}+c_{22} \sigma_{1} \sigma_{2} \mathbf{h}_{1}^{H} \mathbf{h}_{2}+c_{23} \sigma_{1} \sigma_{2} \mathbf{h}_{1}^{H} \mathbf{h}_{3}+\mu_{2} \rho_{12} \\
& +\mu_{3}\left[\rho_{32} \rho_{31}-\rho_{12}\right]=0, \\
& c_{31} \sigma_{1} \sigma_{3}\left|\mathbf{h}_{1}\right|^{2}+c_{32} \sigma_{1} \sigma_{3} \mathbf{h}_{1}^{H} \mathbf{h}_{2}+c_{32} \sigma_{1} \sigma_{3} \mathbf{h}_{1}^{H} \mathbf{h}_{3}+\mu_{4} \rho_{13} \\
& +\mu_{3}\left[\rho_{12} \rho_{23}-\rho_{13}\right]=0,
\end{aligned}
$$

$$
\begin{aligned}
& c_{31} \sigma_{2} \sigma_{3} \mathbf{h}_{2}^{H} \mathbf{h}_{1}+c_{32} \sigma_{2} \sigma_{3}\left|\mathbf{h}_{2}\right|^{2}+c_{32} \sigma_{2} \sigma_{3} \mathbf{h}_{2}^{H} \mathbf{h}_{3}+\mu_{5} \rho_{23} \\
& +\mu_{3}\left[\rho_{21} \rho_{13}-\rho_{23}\right]=0,
\end{aligned}
$$

where $c_{i j}$ denotes the $(i, j)$ entry of $\operatorname{Adj}\left(\left(I_{3}+Q H^{H} H\right)\right)$ and are given in Appendix B, and

$\begin{cases}\mu_{3}=0 & \text { if } 1+2 \Re\left[\rho_{12} \rho_{23} \rho_{31}\right]-\left|\rho_{12}\right|^{2}-\left|\rho_{31}\right|^{2}-\left|\rho_{23}\right|^{2} \geq 0, \\ \mu_{3}>0 & \text { if } 1+2 \Re\left[\rho_{12} \rho_{23} \rho_{31}\right]-\left|\rho_{12}\right|^{2}-\left|\rho_{31}\right|^{2}-\left|\rho_{23}\right|^{2}<0,\end{cases}$

$$
\left\{\begin{array}{l}
\mu_{4}=0 \quad \text { if }\left|\rho_{13}\right|^{2}<1, \\
\mu_{4}>0 \quad \text { if }\left|\rho_{13}\right|^{2}=1,
\end{array}\right.
$$

and

$$
\begin{cases}\mu_{5}=0 & \text { if }\left|\rho_{23}\right|^{2}<1 \\ \mu_{5}>0 & \text { if }\left|\rho_{23}\right|^{2}=1\end{cases}
$$

\section{RANDom Channel Matrix With Individual POWER CONSTRAINTS}

It is well-known that the capacity of a flat fading channel with perfect channel state information at the transmitter (CSIT) and perfect channel state information at the receiver (CSIR) is the average of the maximum mutual information for each channel realization $[1,2]$; hence the expression in (4) should apply for each fading state. On the other hand, with perfect CSIR only, the ergodic capacity is given as [6]

$$
C=\max _{Q \succeq 0, Q_{i i}=p_{i}} E_{\mathbf{H}}\left[\log \operatorname{det}\left[I_{2}+Q \mathbf{H}^{H} \mathbf{H}\right]\right] .
$$


The optimal $Q$ is dependent on the stationary distribution of the channel process [2]. One way to approximate the capacity is to optimize for the Jensen's upper-bound on the mutual information obtained by using Jensen's inequality [10] as

$$
E_{\mathbf{H}}\left[\log \operatorname{det}\left[I_{2}+Q \mathbf{H}^{H} \mathbf{H}\right]\right] \leq \log \operatorname{det}\left[I_{2}+Q E_{\mathbf{H}}\left[\mathbf{H}^{H} \mathbf{H}\right]\right] \text {. }
$$

Then similar to the expression in (4), we may express the optimal input correlation coefficient for $L=2$ as

$$
\begin{aligned}
& \rho_{12}=\frac{E\left[\mathbf{h}_{1}^{H} \mathbf{h}_{\mathbf{2}}\right]}{\sqrt{\frac{P^{2}}{4}-\Delta_{2}^{2}}\left[E\left|\mathbf{h}_{1}\right|^{2} E\left|\mathbf{h}_{2}\right|^{2}-E\left[\left|\mathbf{h}_{1}^{H} \mathbf{h}_{2}\right|^{2}\right]\right]+\mu_{1}}, \\
& \text { for } 0<\Delta_{2}<\frac{P}{2} \text { and } \mathbf{h}_{1}^{H} \mathbf{h}_{2} \neq 0 .
\end{aligned}
$$

Moreover, an analogous form of Proposition 1 will follow.

\section{CONCLUSION}

In this letter, the design of the optimal input covariance matrix in a Gaussian MIMO channel with both common and individual power constraints is considered. Carrying out the optimization analytically using the standard Lagrangian approach has led to an explicit expression, in terms of the difference between the allocated powers and the channel correlations, for the range of input SNR for which beamforming is optimal in TIMO channels with individual power constraints. The results are extended to random channels using Jensen's upper bound on mutual information. The obtained results are relevant to a number of MIMO set-ups such as distributed MIMO systems.

\section{APPENDIX A}

The determinant of $\left[I_{2}+Q H^{H} H\right]$ can be written as

$$
\begin{aligned}
& \operatorname{det}\left[I_{2}+H^{H} H Q\right]=1+\sigma_{1}^{2}\left|\mathbf{h}_{1}\right|^{2}+\sigma_{2}^{2}\left|\mathbf{h}_{2}\right|^{2} \\
& +\sigma_{1} \sigma_{2}\left[\rho_{12} \mathbf{h}_{2}^{H} \mathbf{h}_{1}+\rho_{21} \mathbf{h}_{1}^{H} \mathbf{h}_{2}\right] \\
& +\sigma_{1}^{2} \sigma_{2}^{2}\left[\left|\mathbf{h}_{1}\right|^{2}\left|\mathbf{h}_{2}\right|^{2}-\left|\mathbf{h}_{1}^{H} \mathbf{h}_{2}\right|^{2}\right]\left[1-\rho_{12} \rho_{21}\right] .
\end{aligned}
$$

Since the logarithm function can be maximized by maximizing its argument [11, p. 278], we may to derive the optimal $Q$ by considering the following reduced problem,

$$
\begin{aligned}
& \max _{Q \succeq 0} \operatorname{det}\left[I_{2}+Q H^{H} H\right], \\
& \text { s.t. } \sigma_{1}^{2}+\sigma_{2}^{2}=P, \sigma_{1}^{2} \geq 0, \sigma_{2}^{2} \geq 0, \text { and }\left|\rho_{12}\right|^{2} \leq 1 .
\end{aligned}
$$

Using the Karush-Kuhn-Tucker conditions for the optimization problem, we get

$$
\begin{aligned}
& \sigma_{1}^{2}\left[\left|\mathbf{h}_{1}\right|^{2}+\sigma_{2}^{2}\left[\left|\mathbf{h}_{1}\right|^{2}\left|\mathbf{h}_{2}\right|^{2}-\left|\mathbf{h}_{1}^{H} \mathbf{h}_{2}\right|^{2}\right]\left(1-\rho_{12} \rho_{21}\right)-\lambda\right] \\
& +\sigma_{1} \sigma_{2} \Re\left[\rho_{12} \mathbf{h}_{2}^{H} \mathbf{h}_{1}\right]=0, \\
& \sigma_{2}^{2}\left[\left|\mathbf{h}_{2}\right|^{2}+\sigma_{1}^{2}\left[\left|\mathbf{h}_{1}\right|^{2}\left|\mathbf{h}_{2}\right|^{2}-\left|\mathbf{h}_{1}^{H} \mathbf{h}_{2}\right|^{2}\right]\left(1-\rho_{12} \rho_{21}\right)-\lambda\right] \\
& +\sigma_{1} \sigma_{2} \Re\left[\rho_{12} \mathbf{h}_{2}^{H} \mathbf{h}_{1}\right]=0,
\end{aligned}
$$

and

$$
\rho_{12}=\frac{\mathbf{h}_{1}^{H} \mathbf{h}_{2}}{\sigma_{1} \sigma_{2}\left[\left|\mathbf{h}_{1}\right|^{2}\left|\mathbf{h}_{2}\right|^{2}-\left|\mathbf{h}_{1}^{H} \mathbf{h}_{2}\right|^{2}\right]+\mu_{1}},
$$

$$
\rho_{21}=\rho_{12}^{*}
$$

where

$$
\begin{cases}\mu_{1}=0 & \text { if }\left|\rho_{12}\right|^{2}<1 \\ \mu_{1}>0 & \text { if }\left|\rho_{12}\right|^{2}=1\end{cases}
$$

and $\lambda$ is the Lagrange multiplier associated with the equality constraint. Note that the non-negativity constraint for $\sigma_{1}$ and $\sigma_{2}$ is implicitly included in the expressions in (20a) and (20b). First, we observe that the optimal $\sigma_{1}$ and $\sigma_{2}$ have to be non-zero as far as $\mathbf{h}_{1}^{H} \mathbf{h}_{2} \neq 0$ which can be seen from the expressions in (18) and (21a). So, if we set $\sigma_{1}^{2}=\frac{P}{2}+\Delta$ and $\sigma_{2}^{2}=\frac{P}{2}-\Delta$, where $\Delta<\frac{P}{2}$, then the expression in (4) will follow from (21a). Furthermore, since $\mu_{1}>0$ for $\left|\rho_{12}\right|=1$ (i.e., beamforming takes place), the expression in (5) will follow.

Second, for MIMO channels with a common power constraint, the expressions in (20a) and (20b) are involved to solve for $\sigma_{1}$ and $\sigma_{2}$, and determine $\Delta$; instead, we propose the following solution: we may first solve for $\sigma_{1}$ and $\sigma_{2}$ (by setting $\left.\left|\rho_{12}\right|=0\right)$ and then compute the corresponding value $\rho_{12}$ using (21a); however if the magnitude of $\rho_{12}$ becomes unity or one of the allocated powers comes out to be zero (which will violate the observation stated above), then we go back to (20a) and (20b) to solve for $\sigma_{1}$ and $\sigma_{2}$ with $\left|\rho_{12}\right|=1$. The intuition behind this solution is that as far as beamforming is not the optimal transmission strategy, we may consider two uncorrelated Gaussian inputs, determine the optimal allocated powers, and then introduce the optimal correlation between them. So, setting $\left|\rho_{12}\right|=0$ in (20a) and (20b) results in

$$
\begin{array}{r}
\left|\mathbf{h}_{1}\right|^{2}+\sigma_{2}^{2}\left[\left|\mathbf{h}_{1}\right|^{2}\left|\mathbf{h}_{2}\right|^{2}-\left|\mathbf{h}_{1}^{H} \mathbf{h}_{2}\right|^{2}\right]-\lambda=0, \\
\left|\mathbf{h}_{2}\right|^{2}+\sigma_{1}^{2}\left[\left|\mathbf{h}_{1}\right|^{2}\left|\mathbf{h}_{2}\right|^{2}-\left|\mathbf{h}_{1}^{H} \mathbf{h}_{2}\right|^{2}\right]-\lambda=0,
\end{array}
$$

we may get $\sigma_{1}^{2}=\frac{1}{2}\left[\frac{\left|\mathbf{h}_{1}\right|^{2}-\left|\mathbf{h}_{2}\right|^{2}}{\left[\left|\mathbf{h}_{1}\right|^{2}\left|\mathbf{h}_{2}\right|^{2}-\left|\mathbf{h}_{1}^{H} \mathbf{h}_{2}\right|^{2}\right]}+P\right]$ and $\sigma_{2}^{2}=$ $\frac{1}{2}\left[\frac{\left|\mathbf{h}_{2}\right|^{2}-\left|\mathbf{h}_{1}\right|^{2}}{\left.\left.|| \mathbf{h}_{1}\right|^{2}\left|\mathbf{h}_{2}\right|^{2}-\left|\mathbf{h}_{1}^{H} \mathbf{h}_{2}\right|^{2}\right]}+P\right]$. However, since $0<\sigma_{1}^{2}<P$ and $0<\sigma_{2}^{2}<P$, then based on these expressions, we may define

$$
\Delta_{1}=\frac{1}{2} \frac{\left|\mathbf{h}_{1}\right|^{2}-\left|\mathbf{h}_{2}\right|^{2}}{\left[\left|\mathbf{h}_{1}\right|^{2}\left|\mathbf{h}_{2}\right|^{2}-\left|\mathbf{h}_{1}^{H} \mathbf{h}_{2}\right|^{2}\right]},
$$

Now if $\Delta_{1}=\frac{P}{2}$, then a violation will take place since either $\sigma_{1}$ or $\sigma_{2}$ would be zero. The candidate solution is to set $\left|\rho_{12}\right|$ to unity and compute the corresponding optimal values of the allocated powers. Substituting $\left|\rho_{12}\right|=1$ in (20a) and (20b) results in

$$
\begin{aligned}
\sigma_{1}^{2}\left[\left|\mathbf{h}_{1}\right|^{2}-\lambda\right] & =-\sigma_{1} \sigma_{2} \Re\left[\rho_{12} \mathbf{h}_{2}^{H} \mathbf{h}_{1}\right], \\
\sigma_{2}^{2}\left[\left|\mathbf{h}_{2}\right|^{2}-\lambda\right] & =-\sigma_{1} \sigma_{2} \Re\left[\rho_{12} \mathbf{h}_{2}^{H} \mathbf{h}_{1}\right] .
\end{aligned}
$$

Using (24a) and (24b), we may get $\frac{\sigma_{1}^{2}-\sigma_{2}^{2}}{\sigma_{1} \sigma_{2}}=\frac{\left|\mathbf{h}_{1}\right|^{2}-\left|\mathbf{h}_{2}\right|^{2}}{\Re\left[\rho_{12} \mathbf{h}_{2}^{H} \mathbf{h}_{1}\right]}$ which can be solved numerically for $\sigma_{1}^{2}$ and $\sigma_{2}^{2}$.

\section{APPENDIX B}

The $c_{i j}$ expressions are given as in (25)-(30). 


$$
\begin{aligned}
c_{21}= & \sigma_{1}^{2} \sigma_{2} \sigma_{3}\left[\rho_{31} \rho_{12}\left[\mathbf{h}_{1}^{H} \mathbf{h}_{2} \mathbf{h}_{2}^{H} \mathbf{h}_{3}-\mathbf{h}_{1}^{H} \mathbf{h}_{3}\left|\mathbf{h}_{2}\right|^{2}\right]+\rho_{32}\left[\mathbf{h}_{1}^{H} \mathbf{h}_{3}\left|\mathbf{h}_{2}\right|^{2}-\mathbf{h}_{1}^{H} \mathbf{h}_{2} \mathbf{h}_{2}^{H} \mathbf{h}_{3}\right]\right] \\
& +\sigma_{1}^{2} \sigma_{3}^{2}\left[\mathbf{h}_{3}^{H} \mathbf{h}_{2} \mathbf{h}_{1}^{H} \mathbf{h}_{3}-\mathbf{h}_{1}^{H} \mathbf{h}_{2}\left|\mathbf{h}_{3}\right|^{2}+\left|\rho_{13}\right|^{2}\left[\mathbf{h}_{1}^{H} \mathbf{h}_{2}\left|\mathbf{h}_{3}\right|^{2}-\mathbf{h}_{3}^{H} \mathbf{h}_{2} \mathbf{h}_{1}^{H} \mathbf{h}_{3}\right]\right] \\
& +\sigma_{1} \sigma_{2} \sigma_{3}^{2}\left[\rho_{12}\left[\left|\mathbf{h}_{2}^{H} \mathbf{h}_{3}\right|^{2}-\left|\mathbf{h}_{2}\right|^{2}\left|\mathbf{h}_{3}\right|^{2}\right]+\rho_{32} \rho_{13}\left[\left|\mathbf{h}_{2}\right|^{2}\left|\mathbf{h}_{3}\right|^{2}-\left|\mathbf{h}_{2}^{H} \mathbf{h}_{3}\right|^{2}\right]\right] \\
& -\sigma_{1}^{2} \mathbf{h}_{1}^{H} \mathbf{h}_{2}-\rho_{12} \sigma_{1} \sigma_{2}\left|\mathbf{h}_{2}\right|^{2}-\rho_{13} \sigma_{1} \sigma_{3} \mathbf{h}_{3}^{H} \mathbf{h}_{2},
\end{aligned}
$$

$$
\begin{aligned}
c_{22}= & 1+\left.\sigma_{1}^{2}\left|\mathbf{h}_{1}\right|^{2}\left|+\rho_{12} \sigma_{1} \sigma_{2} \mathbf{h}_{2}^{H} \mathbf{h}_{1}+\rho_{31} \sigma_{1} \sigma_{3} \mathbf{h}_{1}^{H} \mathbf{h}_{3}+\rho_{13} \sigma_{1} \sigma_{3} \mathbf{h}_{3}^{H} \mathbf{h}_{1}+\sigma_{3}^{2}\right| \mathbf{h}_{3}\right|^{2} \mid+\rho_{32} \sigma_{2} \sigma_{3} \mathbf{h}_{2}^{H} \mathbf{h}_{3} \\
& +\sigma_{1}^{2} \sigma_{2} \sigma_{3}\left[\rho_{31} \rho_{12}\left[\mathbf{h}_{2}^{H} \mathbf{h}_{1} \mathbf{h}_{1}^{H} \mathbf{h}_{3}-\mathbf{h}_{2}^{H} \mathbf{h}_{3}\left|\mathbf{h}_{1}\right|^{2}\right]+\rho_{32}\left[\mathbf{h}_{2}^{H} \mathbf{h}_{3}\left|\mathbf{h}_{1}\right|^{2}-\mathbf{h}_{1}^{H} \mathbf{h}_{3} \mathbf{h}_{2}^{H} \mathbf{h}_{1}\right]\right] \\
& +\sigma_{1}^{2} \sigma_{3}^{2}\left[\left|\mathbf{h}_{1}\right|^{2}\left|\mathbf{h}_{3}\right|^{2}-\left|\mathbf{h}_{1}^{H} \mathbf{h}_{3}\right|^{2}+\left|\rho_{13}\right|^{2}\left[\mathbf{h}_{3}^{H} \mathbf{h}_{1} \mathbf{h}_{1}^{H} \mathbf{h}_{2}-\left|\mathbf{h}_{1}\right|^{2}\left|\mathbf{h}_{3}\right|^{2}\right]\right] \\
& +\sigma_{1} \sigma_{2} \sigma_{3}^{2}\left[\rho_{12}\left[\mathbf{h}_{2}^{H} \mathbf{h}_{1}\left|\mathbf{h}_{3}\right|^{2}-\mathbf{h}_{2}^{H} \mathbf{h}_{3} \mathbf{h}_{3}^{H} \mathbf{h}_{1}\right]+\rho_{32} \rho_{13}\left[\mathbf{h}_{3}^{H} \mathbf{h}_{1} \mathbf{h}_{2}^{H} \mathbf{h}_{3}\right]\right],
\end{aligned}
$$

$$
\begin{aligned}
c_{23}= & \sigma_{1}^{2} \sigma_{2} \sigma_{3}\left[\rho_{32}\left[\left|\mathbf{h}_{1}^{H} \mathbf{h}_{2}\right|^{2}-\left|\mathbf{h}_{1}\right|^{2}\left|\mathbf{h}_{2}\right|^{2}\right]+\rho_{12} \rho_{31}\left[\left|\mathbf{h}_{1}\right|^{2}\left|\mathbf{h}_{2}\right|^{2}-\left|\mathbf{h}_{1}^{H} \mathbf{h}_{2}\right|^{2}\right]\right] \\
& +\sigma_{1}^{2} \sigma_{3}^{2}\left[\mathbf{h}_{1}^{H} \mathbf{h}_{2} \mathbf{h}_{3}^{H} \mathbf{h}_{1}-\mathbf{h}_{3}^{H} \mathbf{h}_{2}\left|\mathbf{h}_{1}\right|^{2}+\left|\rho_{13}\right|^{2}\left[\mathbf{h}_{3}^{H} \mathbf{h}_{2}\left|\mathbf{h}_{1}\right|^{2}-\mathbf{h}_{1}^{H} \mathbf{h}_{2} \mathbf{h}_{3}^{H} \mathbf{h}_{1}\right]\right] \\
& +\sigma_{1} \sigma_{2} \sigma_{3}^{2}\left[\rho_{12}\left[\mathbf{h}_{3}^{H} \mathbf{h}_{2}\left|\mathbf{h}_{2}\right|^{2}-\mathbf{h}_{2}^{H} \mathbf{h}_{1} \mathbf{h}_{3}^{H} \mathbf{h}_{2}\right]+\rho_{32} \rho_{13}\left[\mathbf{h}_{3}^{H} \mathbf{h}_{2} \mathbf{h}_{2}^{H} \mathbf{h}_{1}-\mathbf{h}_{3}^{H} \mathbf{h}_{1}\left|\mathbf{h}_{2}\right|^{2}\right]\right] \\
& -\rho_{13} \sigma_{1} \sigma_{2} \mathbf{h}_{1}^{H} \mathbf{h}_{2}-\rho_{32} \sigma_{2} \sigma_{3}\left|\mathbf{h}_{2}\right|^{2}-\sigma_{3}^{2} \mathbf{h}_{3}^{H} \mathbf{h}_{2},
\end{aligned}
$$

$$
\begin{aligned}
c_{31}= & \sigma_{1}^{2} \sigma_{2} \sigma_{3}\left[\rho_{13} \rho_{21}\left[\mathbf{h}_{3}^{H} \mathbf{h}_{2} \mathbf{h}_{1}^{H} \mathbf{h}_{3}-\mathbf{h}_{1}^{H} \mathbf{h}_{2}\left|\mathbf{h}_{3}\right|^{2}\right]+\rho_{32}\left[\mathbf{h}_{2}^{H} \mathbf{h}_{3}\left|\mathbf{h}_{1}\right|^{2}-\mathbf{h}_{1}^{H} \mathbf{h}_{3} \mathbf{h}_{2}^{H} \mathbf{h}_{1}\right]\right] \\
& +\sigma_{1}^{2} \sigma_{2}^{2}\left[\mathbf{h}_{1}^{H} \mathbf{h}_{2} \mathbf{h}_{2}^{H} \mathbf{h}_{3}-\mathbf{h}_{1}^{H} \mathbf{h}_{3}\left|\mathbf{h}_{2}\right|^{2}+\left|\rho_{12}\right|^{2}\left[\mathbf{h}_{1}^{H} \mathbf{h}_{3}\left|\mathbf{h}_{2}\right|^{2}-\mathbf{h}_{2}^{H} \mathbf{h}_{3} \mathbf{h}_{1}^{H} \mathbf{h}_{3}\right]\right] \\
& +\sigma_{1} \sigma_{2}^{2} \sigma_{3}\left[\rho_{13}\left[\left|\mathbf{h}_{2}^{H} \mathbf{h}_{3}\right|^{2}-\left|\mathbf{h}_{2}\right|^{2}\left|\mathbf{h}_{3}\right|^{2}\right]+\rho_{12} \rho_{23}\left[\left|\mathbf{h}_{2}\right|^{2}\left|\mathbf{h}_{3}\right|^{2}-\left|\mathbf{h}_{2}^{H} \mathbf{h}_{3}\right|^{2}\right]\right]
\end{aligned}
$$

$$
\begin{aligned}
c_{32}= & \sigma_{1}^{2} \sigma_{2} \sigma_{3}\left[\rho_{13} \rho_{21}\left[\left.\mathbf{h}_{1}\right|^{2}\left|\mathbf{h}_{3}\right|^{2}-\left|\mathbf{h}_{1}^{H} \mathbf{h}_{3}\right|^{2}\right]+\rho_{32}\left[\left|\mathbf{h}_{1}^{H} \mathbf{h}_{3}\right|^{2}-\left|\mathbf{h}_{1}\right|^{2}\left|\mathbf{h}_{3}\right|^{2}\right]\right] \\
& +\sigma_{1}^{2} \sigma_{2}^{2}\left[\mathbf{h}_{2}^{H} \mathbf{h}_{1} \mathbf{h}_{1}^{H} \mathbf{h}_{3}-\mathbf{h}_{2}^{H} \mathbf{h}_{3}\left|\mathbf{h}_{1}\right|^{2}+\left|\rho_{12}\right|^{2}\left[\mathbf{h}_{2}^{H} \mathbf{h}_{3}\left|\mathbf{h}_{1}\right|^{2}-\mathbf{h}_{2}^{H} \mathbf{h}_{1} \mathbf{h}_{1}^{H} \mathbf{h}_{3}\right]\right] \\
& +\sigma_{1} \sigma_{2}^{2} \sigma_{3}\left[\rho_{13}\left[\mathbf{h}_{2}^{H} \mathbf{h}_{1}\left|\mathbf{h}_{3}\right|^{2}-\mathbf{h}_{3}^{H} \mathbf{h}_{1} \mathbf{h}_{2}^{H} \mathbf{h}_{3}\right]+\rho_{12} \rho_{23}\left[\mathbf{h}_{3}^{H} \mathbf{h}_{1} \mathbf{h}_{2}^{H} \mathbf{h}_{3}-\mathbf{h}_{2}^{H} \mathbf{h}_{1}\left|\mathbf{h}_{3}\right|^{2}\right]\right] \\
& -\rho_{12} \sigma_{1} \sigma_{2} \mathbf{h}_{1}^{H} \mathbf{h}_{3}-\sigma_{2}^{2} \mathbf{h}_{2}^{H} \mathbf{h}_{3}-\rho_{12} \sigma_{1} \sigma_{2}\left|\mathbf{h}_{3}\right|^{2},
\end{aligned}
$$

$$
\begin{aligned}
c_{33}= & +\left.\sigma_{1}^{2}\left|\mathbf{h}_{1}\right|^{2}\left|+\rho_{12} \sigma_{1} \sigma_{2} \mathbf{h}_{2}^{H} \mathbf{h}_{1}+\rho_{13} \sigma_{1} \sigma_{3} \mathbf{h}_{3}^{H} \mathbf{h}_{1}+\rho_{21} \sigma_{1} \sigma_{2} \mathbf{h}_{1}^{H} \mathbf{h}_{2} \sigma_{2}^{2}\right| \mathbf{h}_{2}\right|^{2} \mid+\rho_{23} \sigma_{2} \sigma_{3} \mathbf{h}_{3}^{H} \mathbf{h}_{2} \\
& +\sigma_{1}^{2} \sigma_{2} \sigma_{3}\left[\rho_{13} \rho_{21}\left[\mathbf{h}_{3}^{H} \mathbf{h}_{1} \mathbf{h}_{1}^{H} \mathbf{h}_{2}-\mathbf{h}_{3}^{H} \mathbf{h}_{2}\left|\mathbf{h}_{1}\right|^{2}\right]+\rho_{32}\left[\mathbf{h}_{3}^{H} \mathbf{h}_{2}\left|\mathbf{h}_{1}\right|^{2}-\mathbf{h}_{3}^{H} \mathbf{h}_{1} \mathbf{h}_{1}^{H} \mathbf{h}_{2}\right]\right] \\
& +\sigma_{1}^{2} \sigma_{2}^{2}\left[\left|\mathbf{h}_{1}\right|^{2}\left|\mathbf{h}_{2}\right|^{2}-\left|\mathbf{h}_{1}^{H} \mathbf{h}_{2}\right|^{2}+\left|\rho_{12}\right|^{2}\left[\left|\mathbf{h}_{1}^{H} \mathbf{h}_{2}\right|^{2}-\left|\mathbf{h}_{1}\right|^{2}\left|\mathbf{h}_{2}\right|^{2}\right]\right] \\
& +\sigma_{1} \sigma_{2}^{2} \sigma_{3}\left[\rho_{13}\left[\mathbf{h}_{3}^{H} \mathbf{h}_{1}\left|\mathbf{h}_{2}\right|^{2}-\mathbf{h}_{2}^{H} \mathbf{h}_{1} \mathbf{h}_{3}^{H} \mathbf{h}_{2}\right]+\rho_{12} \rho_{23}\left[\mathbf{h}_{2}^{H} \mathbf{h}_{1} \mathbf{h}_{3}^{H} \mathbf{h}_{2}-\mathbf{h}_{3}^{H} \mathbf{h}_{1}\left|\mathbf{h}_{2}\right|^{2}\right]\right] .
\end{aligned}
$$

\section{REFERENCES}

[1] A. Goldsmith, S. A. Jafar, N. Jindal, and S. Vishwanath, "Capacity limits in MIMO channels," IEEE J. Sel. Areas Commun., vol. 21, no. 5, pp. 684-702, June 2003.

[2] D. N. C. Tse and P. Viswanath, Fundamentals of Wireless Communications. Cambridge University Press, 2005.

[3] W. Choi and J. G. Andrews, "Downlink performance and capacity of distributed antenna systems in a multicell environment," IEEE Trans. Wireless Commun., vol. 6, no. 1, pp. 69-73, Jan. 2007.

[4] H. Hu, Y. Zhang, and J. Luo (editors), Distributed Antenna Systems: Open Architecture for Future Wireless Communications. CRC Press, 2007.

[5] X. Zheng, Y. Xie, J. Li, and P. Stoica, "MIMO transmit beamforming under uniform elemental power constraint," IEEE Trans. Signal Process., vol. 55, no. 11, pp. 5395-5406, Nov. 2007.
[6] I. E. Telatar, "Capacity of multi-antenna Gaussian channel," Euro. Trans. Telecommun., vol. 10, pp. 585-595, Nov. 1999.

[7] F. Nesser and J. Massey, "Proper complex random processes with applications to information theory," IEEE. Trans. Inf. Theory, vol. 39, no. 4, pp. 1293-1302, July 1993.

[8] J. Bach Andersen, "Array gain and capacity for known random channels with multiple element arrays at both ends," IEEE J. Sel. Areas Commun., vol. 18 , no. 11 , pp. $2172-2178$, Nov. 2000,

[9] K. N. Swamy, "On Sylvester's criterion for positive-semidefinite matrices," IEEE. Trans. Automat. Contr., vol. 18, no. 3, pp. 306-306, June 1973.

[10] M. Vu and A. Paulraj, "On the capacity of MIMO wireless channels with dynamic CSIT," IEEE J. Sel. Areas Commun., vol. 25, no. 7, pp. 1269-1283, Sep. 2007.

[11] T. M. Cover and J. A. Thomas, Elements of Information Theory, 2nd edition. John Wiley \& Sons, Inc., 2006. 